\title{
A Review on Image Enhancement Methods
}

\author{
Ashwini R. Karande \\ shivaji university, Kolhapur \\ Dept. Of Electronics, \\ KIT's collage of Engineering, \\ Kolhapur, Maharashtra.
}

\author{
A. L. Renke \\ Asst.prof. \\ shivaji university, Kolhapur \\ Dept. Of Electronics, \\ KIT's collage of Engineering, \\ Kolhapur, Maharashtra.
}

\begin{abstract}
Image enhancement is very important in the field where visual appearance of an image is the main. Image enhancement is the process of improving the image in such a way that the resulting or output image is more suitable than the original image for specific task. With the help of image enhancement process the quality of image can be improved to get good quality images so that they can be clear for human perception or for the further analysis done by machines. Processing of satellite images is very much essential in the computer science research field. The image taken by satellites may be affected by many reasons such as climate, weather and other factors. Important information may not be clear because of blurred or not so clear images. Satellite images have complete information but may not visible, that information can be improved by image enhancement. Satellite images are used in many fields such as astronomy, geographical information system and geoscience studies. Image enhancement method enhances the quality, visual appearance, improves clarity of images, removes blurring and noise, increases contrast and reveals details. The aim of this paper is to study and determine limitations of the existing IE techniques. This paper will provide an overview of different IE techniques commonly used.
\end{abstract}

\section{Keywords}

Image enhancement, Histogram equalization, Discrete wavelet transform(DWT), Wavelet zero padding(WZP), Undecimented wavelet transform (UWT), Dual Tree- Complex Wavelet Transform (DT-CWT).

\section{INTRODUCTION}

Image enhancement is the process of improving the quality of digital image whatever may be the source of degradation. IE is the main technique for improving the resolution and visual appearance of the image where the experts make decision on the basis of the image information. IE cannot increase the data or information content in the image, it can only improve the dynamic range and highlight certain features of interest in image. IE technique can be divided into two broad categories.

\section{A) Spatial Domain Enhancement Method}

The term spatial domain refers to the image plane i.e. the collection of pixels composing an image. Spatial domain techniques are the procedures that operate directly on these pixels. To achieve enhancement, pixel values are directly manipulated.

B) Frequency domain enhancement method

Here, transferring the image into frequency domain is done by the Fourier transform of the image to be enhanced. All the enhancement operations required are carried out on that Fourier transform and inverse transform is taken to produce the final enhanced image.

Satellite images can capture a lot of information, but they have issues with their resolution and also appeared as blurred. These all issues related with satellite images make enhancement more necessary so that the visibility of images gets improved and all unwanted noise get removed. IE helps in extracting useful information from satellite images. These satellite images are used for weather forecast, crop production forecast, military intelligence and metrology, disaster mitigation planning and recovery and many more. So the enhancement of these images is very much necessary.

\section{IMAGE ENHANCEMENT TECHNIQUES: \\ 2.1 Histogram equalization}

Histogram shows the distribution of data in the image graphically. How many times a Specific gray level intensities appears in an image is shown in histogram.

Equalization of histogram is a method that enhances and adjusts the contrast in an image. It is the effective and simple method of improving quality of image. HE method improves the global contrast of image where the usable data with the image is represented by close contrast values. With this method the intensities are better distributed within the histogram.

\subsection{Gray level transformation}

Basic gray level transformations are as below.

- Linear

- Logarithmic

- $\quad$ Power - law

Linear transformation

Linear transformation is divided into two types simple identity and negative transformation. 


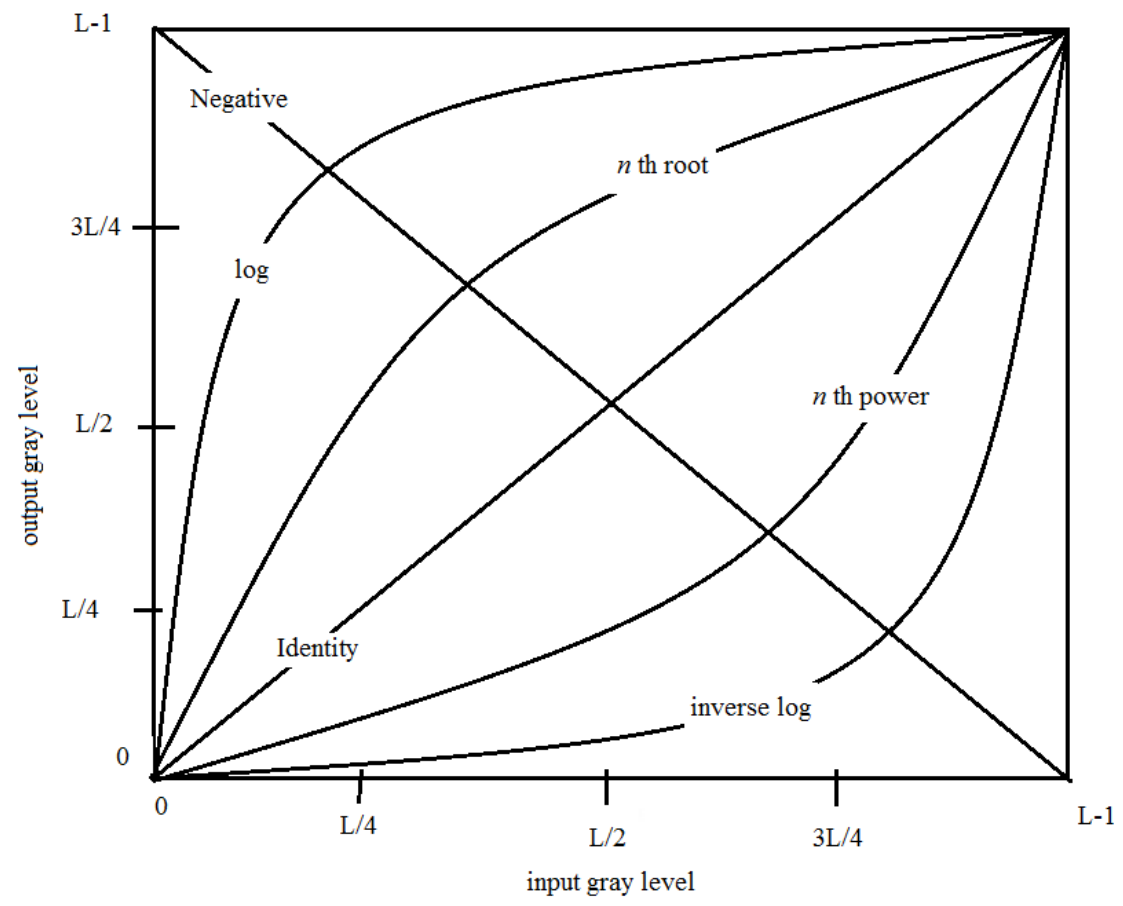

Fig1. Plot of various transformation functions

\section{Identity transformation}

Here, each value of the input image is directly mapped to each other value of output image. As a result of this, we get the same input image and output image. Hence the name is identity transformation.

\section{Negative transformation}

In this each value of the input image is subtracted from the L-1 and mapped onto the output image.

\section{Log transformation}

Here, a narrow range of low level input grey scale intensities into a wider range of output values.

Log transformations can be given by the formula,

$\mathrm{S}=\mathrm{c} \cdot \log (1+\mathrm{r})$

Here, $s$ and $r$ are pixel values of output and input images respectively. $\mathrm{C}$ is a constant. 1 is added to each pixel value of input image because if $r=0$, then $\log (0)$ will be equal to infinity.In this transformation, dark pixel values get expanded and bright pixel values get compressed.

\section{Power law transformation}

This includes nth power and nth root transformation. The equation for this transformation is given by,

$$
S=C r^{\gamma}
$$

Symbol $\gamma$ is called gamma. Variation in the value of $\gamma$ varies enhancement of the image.

\subsection{Thresholding Transformation}

Thresholding is the easy process of image segmentation through which we can separate an object of our interest from a background. Simple thresholding method replaces an image pixel value below a fixed constant $\mathrm{T}$ with a black pixel and pixel values greater than $\mathrm{T}$ are replaced by a white pixel.

\subsection{Contrast stretching}

It is a process to change pixel brightness values Contrast stretching is an easy method which improves the contrast in an image by stretching the range of intensity values the image contains to a desired range of values.

\subsection{Un-sharp masking}

It is an image sharpening technique. The blurred copy of an image is subtracted from the original image to detect any edges. With the help of these edge details a mask is made. Contrast is increased at the edges and the effect is applied to the original image.

\subsection{Wavelet zero padding (WZP)}

It is very easy method used to enhance an image. In this method a low resolution image is wavelet transformed first and zero matrices are embedded into transformed image. An input low resolution image is used to reconstruct a high resolution image by using zero padding of high frequency sub-bands followed by inverse wavelet transform.

Suppose, $\mathrm{X}$ is an input image of size $\mathrm{m} \mathrm{x} n$, then output image is given as $\mathrm{Y}$

$Y=W^{-1}\left[\begin{array}{cc}X & 0_{m, n} \\ 0_{m, n} & 0_{m, n}\end{array}\right]$

Where, $\boldsymbol{O}_{m, n}$ is an all zero sub matrix of dimension $\mathrm{m} \times \mathrm{n}$ and $W^{-1}$ is the inverse DWT. 


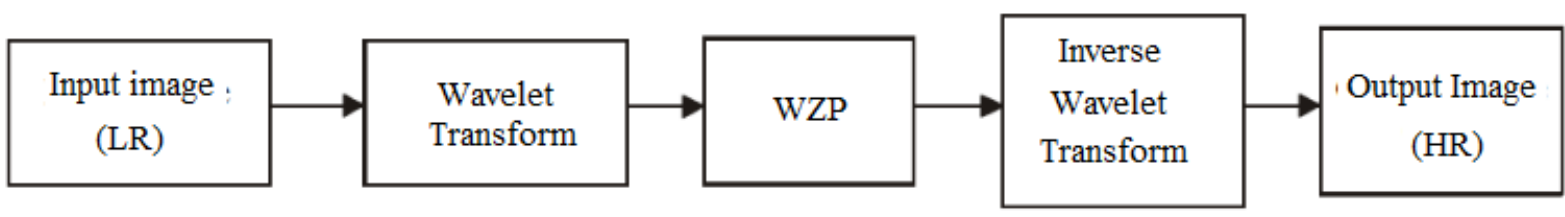

Fig 2: WZP Method

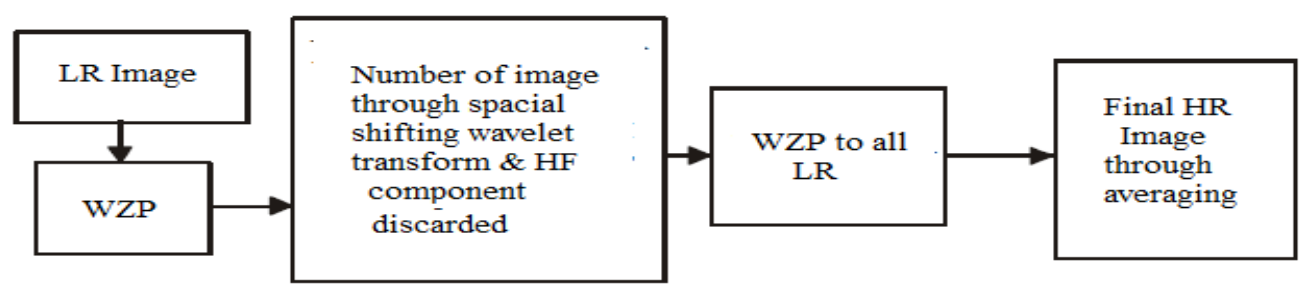

Fig 3: Cycle Spinning

\subsection{Cycle spinning (CS)}

Following steps are followed in this method to get high resolution image.

1. Using WZP method an intermediate High Resolution image is obtained.

2. After this $\mathrm{N}$ number of images are obtained through spatial shifting, wavelet transforming and discarding high frequency component.

3. Again, a number of High Resolution images are obtained by applying WZP process to all Low Resolution images.

4. These High Resolution images are realigned and averaged to give a final High Resolution Output image.

\subsection{Undecimented wavelet transform \\ (UWT)}

In this method, decimation is not used after decomposition of image into different frequency sub bands. First estimate of HR image is obtained by applying WZP.

Suppose, $\mathrm{X}$ is an input LR image of size $\mathrm{m} \mathrm{x} \mathrm{n}$, then estimated HR image is given as $\mathrm{Y}$

$$
\hat{Y}=I D W T\left[\begin{array}{ll}
X & b \\
b & b
\end{array}\right]
$$

Where, $\mathrm{b}$ is zero matrix of size $\mathrm{m} \times \mathrm{n}$ and IDWT is inverse discrete wavelet transform.

Next, UWT is implemented on this estimated HR image, because of this image gets decomposed into two bands called estimated details and approximation coefficients.

These approximation coefficients are replaced by initially estimated HR image.

Finally, inverse UWT is taken to obtain the final HR image.

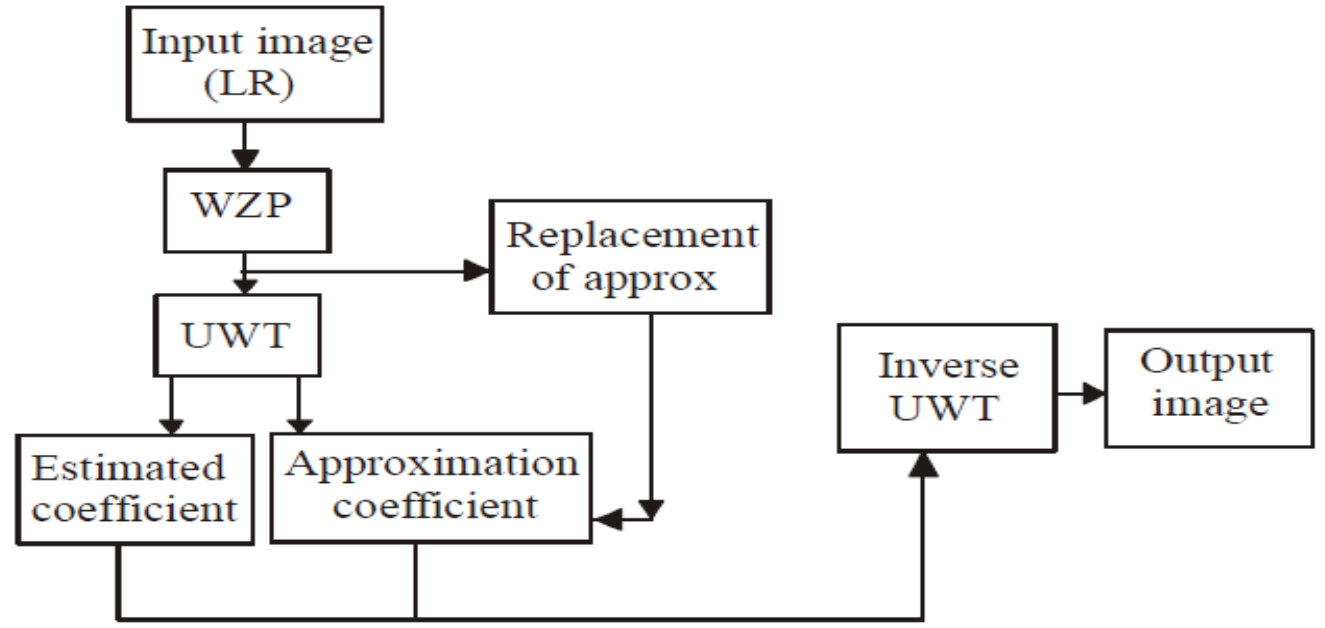

Fig 4: UWT Method 


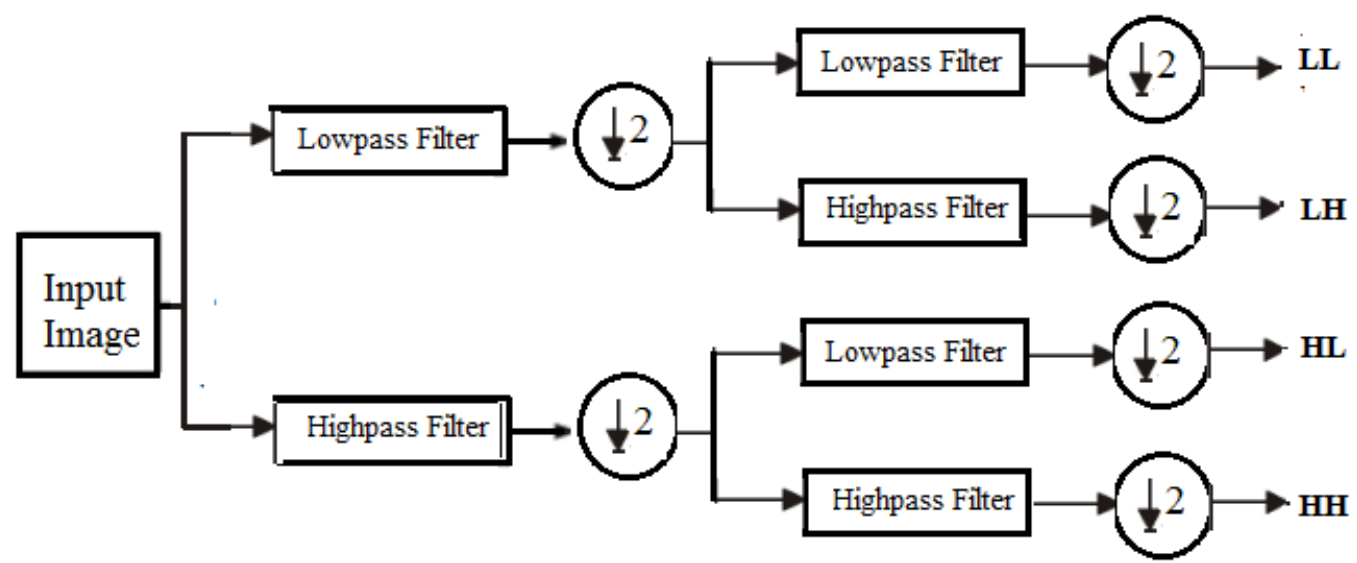

Fig 5: Block Diagram of DWT filter banks of level-I

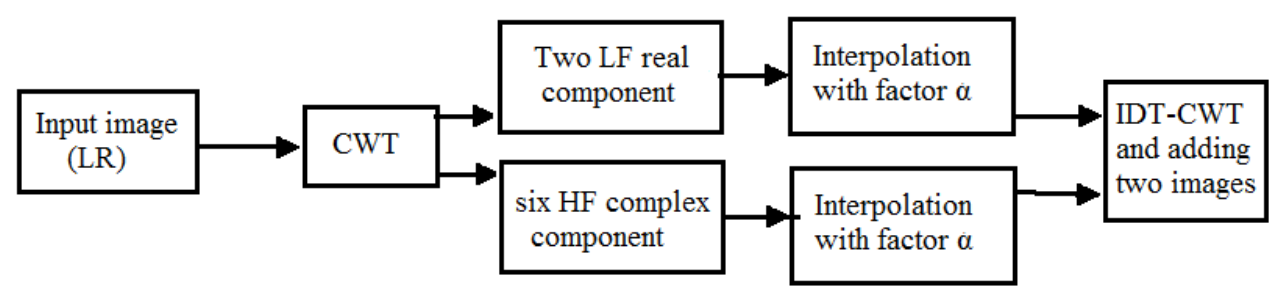

Fig 6: DT-CWT Method

\subsection{Discrete wavelet transform (DWT)}

DWT decomposes a low resolution image into 4 sub band images LL, LH, HL, HH. Then all obtained frequency components (low and high) undergoes interpolation.

By subtracting the interpolated LL image from the original LR image we get a difference image.

After this, difference image and the interpolated high frequency components are added to obtain estimated form of HF sub band images.

Finally, estimated images are combined with the input image using IDWT to obtain High Resolution images.

\subsection{Dual Tree- Complex Wavelet Transform (DT-CWT)}

DT-CWT is used to decompose an input image into different sub band images. Here, direction selective filters are used to generate high-frequency sub band images. They show peak magnitude responses in the presence of image features oriented at $+75^{0},+45^{0},+15^{0},-15^{0},-45^{0},-75^{0}$. The six complex-valued images are interpolated. The interpolated HF sub band images and interpolated input image are combined by using inverse DT-CWT. This gives a high resolution output image.

\section{LITERATURE SURVEY}

Ahmed M. Mahmood, and Jasni Mohmad Zain (2012) did disassembling of Histogram Equalization into building blocks to understand the relationship between Histogram Equalization fundamentals and contrast. Analysing this he determined, with Histogram Equalization we can get changes in density but not in contrast of an image.

Cao, Gang, Yao Zhao, Rongrong Ni, and Xuelong Li.(2014) discovered algorithm to detect the manipulations in digital image where the contrast enhancement is involved.
Cheng, H. D., and Yingtao Zhang.(2012) investigated and analysed the causes of over enhancement. The experimental results shows that the proposed method is good for dynamically monitoring the grade of the entered image.

Chen, Xiaoming, and Lili Lv..(2013) proposed Histogram Equalization based methods combined with multi-scale un-sharp masking based methods. Here HEBM is used to get global contrast enhancement and UMBM is used to get local multi-scale contrast enhancement. The result of this algorithm shows good global as well as local contrast enhancement with noise and artifact suppression.

Reshmalakshmi, C., and M. Sasikumar.(2013) proposed a contrast enhancement algorithm mapping elements from pixel plane to membership plane. Fuzzy sets used in this technique helps to overcome the limitation in existing techniques using contrast enhancement.

Demirel, Hasan, CagriOzcinar, and Gholamreza Anbarjafari.(2010) proposed a contrast enhancement method based on Discrete Wavelet Transform and Singular Value Decomposition for satellite images. Discrete Wavelet Transform (DWT) divides image into four frequency sub-bands and singular value matrix is determined of low low sub-band image and image is reconstructed using Inverse Discrete Wavelet Transform (IDWT).

Maragatham, G., S. MdMansoor Roomi, and T. Manoj Prabu.(2011) Method proposed by them uses Histogram Equalization to find foreground and background pixels of an image and bi-histogram equalization is applied on them. The results shows that this preserves the original image as compared to other techniques.

Arya $p$ unni et al(2014) proposed a method that uses 2D Discrete Wavelet Transform (2D-DWT). This algorithm able to differentiate unnecessary noise contents of the image and high frequency components. Due to possibility of large difference in pixel intensity values the method may not 
maintain average brightness level. It may be under or over saturation.

Turgay Celik And Huseyin Kusetogullari (2009) proposed a method which uses Dual Tree Complex Wavelet Transform (DTCWT) to decompose input image into different frequency sub-bands. Approximate high resolution (HR) image is reconstructed using low resolution LR) image. Zero padding of HF sub-band is done and the output image is constructed by Inverse Complex Wavelet Transform (ICWT).

Hasan Demirel And Gholamreza Anbarjafari (2011) proposed a method to enhance resolution of an image. Using Discrete Wavelet Transform (DWT) input image is decomposed into dissimilar frequency sub-bands. Interpolation of high frequency sub-band image with the input low resolution image is done. With the help of Inverse Discrete Wavelet Transform (IDWT) all these images are combined to get a resolution enhanced image.

Ke, Wei-Ming, Chih-Rung Chen, and Ching-Te Chiu. (2010) says so many image enhancement techniques that exists are used to improve visual appearance of an image. The algorithm proposed by him includes image enhancement framework combining two techniques which are bilateral tone adjustment technique is used to enhance main scenes contained in mid-tone regions. The saliency weighted contrast enhancement combines the concept of image saliency i.e. the property of being noticeable into an easy filter based contrast enhancement technique. This technique enhances the regions very good which may have larger concentration by people.

Chauhan, Ritu, and Sarita Singh Bhadoria. (2011) Histogram Equalization is a technique that is mostly used for contrast enhancement but it has some limitations. Histogram Equalization technique recuperate the incongruity of an image. It alters the intensity level if pixels. Brightness preserving weight clustering histogram equalization gives better result as compared to histogram equalization technique.the brightness preserving weight clustering $\mathrm{HE}$ technique can protect and enhance brightness and visual appearance of an image respectively.

Ghimire, Deepak, and Joonwhoan Lee. (2011) proposed a non linear colour image enhancement technique. The image is enhanced by any technique to get better features and visual appearance. He applied enhancement to only $\mathrm{v}$ components (luminance value) among HSV components of a colour image. According to him $\mathrm{H}$ and $\mathrm{S}$ components don't need modification. The luminance value is enhanced by using non linear transfer function. The function divides $\mathrm{V}$ element into small overlapped blocks and for every pixel within the block luminance enhancement has done. Next, it undergoes contrast enhancement. At the last $\mathrm{H}, \mathrm{S}$ and $\mathrm{V}$ component are converted back to RGB image.

Josephus, ChelsySapna, and S. Remya.(2011) proved the Adaptive Histogram Equalization is best and efficient for the local content significance. But sometimes information get lost and it creates problem for amplification and may introduce speckle noise. Here limited adaptive histogram equalization, the combination of frost and median filter both has been used. Speckle noise in image is removed by the frost filter technique.

Lasmika, K. Raveendra, (2014) proposed method by them is useful for improving quality of satellite image. The proposed method includes DWT which decomposes the input image into different sub-bands and after that it undergoes thresholding. This identifies the area of edges. Morphological filters are used to sharpen the identified edges. The algorithm sharpens and reduces the distortion of an image.

E. Mohan, K.B. Jayarraman, U. Maheswaran, D. Sathiyaraj. G.Dhakshanamoorthi, (2013) proposed a new resolution enhancement technique based on interpolation of the huge frequency sub bands. These huge frequency sub bands obtained by performing DWT on input image. When an image undergoes DWT, the image gets separated into different sub band images. Which are low- low(LL), low-high(LH), high-low(HL) and high-high(HH). with low pass filtering of high resolution image in the wavelet area, the low resolution image is obtained. LL sub band image is used as the input for proposed method. The high frequency sub band image includes high frequency components of image.

S.Venkatramana, S. Narayana Reddy.(2014) proposed a method to improve a resolution of the satellite images based on DWT also includes interpolation 2D-DWT to separate satellite image into LL, LH, HL and HH sub bands. LL sub band preserves the image information and other sub band preserves the edge information. All sub bands undergoes bi-cubic interpolation and IDWT produces the new enhanced output image.

Sundaram, M., K. Ramar, N. Arumugam, and G. Prabin.(2014) proposed a technique based on histogram modified contrast limited adaptive histogram equalization(HE CLAHE). This method is used to regulate level of contrast enhancement. It gives the resultant image having strong contrast and can interpret location detail more relevant. It combines two methods, they are optimization technique and contrast limited adaptive histogram equalization.

Madhu S. Nair. And G. Raju. (2013) proposed method that is based on new fuzzy logic and histogram. This method is used to enhance low contrast colour images. Two parameters M i.e. average intensity value of image that calculated from the histogram and $\mathrm{K}$ i.e. contrast intensification parameter are used in this method. The input RGB image is converted into HSV which preserves the chromatic information in original image. By stretching only $\mathrm{V}$ component under the control of $\mathrm{M}$ and $\mathrm{K}$ parameters the image is enhanced.

Hasan Demirel And Gholamreza Anbarjafari. (2010) in his paper proposed complex wavelet transform (CWT) based image processing technique. Using CWT of an image two complex valued low frequency sub band image, and six complex valued high frequency sub band images are formed. DT-CWT produces dissimilar sub band images using original image. High frequency sub band image undergoes interpolation along with low frequency image. These real valued image are used for the IDT-CWT operation. This also improves the quality and PSNR of the input image and get super resolved output image.

\section{SUMMARY}

The image enhancement techniques are very important in image processing as they play a significant role. These techniques have became very important preprocessing tool. Resolution of image is an important factor. Resolution enhancement helps to create super resolved image of high quality by protecting high frequency components in original image. Image enhancement techniques can be utilized to improve the quality of blurred images. Various image enhancement techniques which exists and are in practice are studied in this paper. Various research papers by different authors also studied. These research papers provide an overview of different enhancement techniques, their 
advantages and limitations or shortcomings. Future research aims at using the experimental study and integration of some of these methods as bases for obtaining an image enhancement technique with greater performance and acceptability in various applications. Among the many image enhancement techniques which are available the choice of procedure depends on utility of image being processed and modality of that image.

\section{REFERENCES}

[1] Ahmed M. Mahmood, and Jasni Mohmad Zain. " A study on the validation of histogram equalization as a contrast enhancement technique". In Advanced Computer Science Applications and Technologies (ACSAT),2012 International Conference on, pp.253-256.IEEE,2012.

[2] Cao, Gang, Yao Zhao, Rongrong Ni, and Xuelong Li. "Contrast enhancement-based forensics in digital images." Information Forensics and Security, IEEE Transactions on 9, no. 3 (2014): 515-525.

[3] Cheng, H. D., and Yingtao Zhang. "Detecting of contrast over-enhancement." In Image Processing (ICIP), 2012 19th IEEE International Conference on, pp. 961-964. IEEE, 2012.

[4] Chen, Xiaoming, and Lili Lv. "A Compositive Contrast Enhancement Algorithm of IR Image." In Information Technology and Applications (ITA), 2013 International Conference on, pp. 58-62. IEEE, 2013.

[5] Reshmalakshmi, C., and M. Sasikumar. "Image contrast enhancement using fuzzy technique." In Circuits, Power and Computing Technologies (ICCPCT), 2013 International Conference on, pp. 861-865. IEEE, 2013.

[6] Demirel, Hasan, CagriOzcinar, and Gholamreza Anbarjafari. "Satellite image contrast enhancement using discrete wavelet transform and singular value decomposition." Geoscience and Remote Sensing Letters, IEEE 7.2: pp.333-337, 2010.

[7] Maragatham, G., S. MdMansoor Roomi, and T. Manoj Prabu. "Contrast enhancement by object based Histogram Equalization." Information and Communication Technologies (WICT), 2011 World Congress on. IEEE, 2011.

[8] Arya P Unni, "Satellite Image Enhancement Using 2D Level DWT", International Journal of Engineering Research \& Technology (IJERT), ISSN: 2278-0181, Vol. 3 Issue 3, March 2014.

[9] Turgay Celik And Huseyin Kusetogullari," Self-Sampled Image Resolution Enhancement Using Dual-Tree Complex Wavelet Transform" In European Signal Processing Conference, Glasgow, Scotland, 2009.

[10] Hasan Demirel And Gholamreza Anbarjafari,"Discrete Wavelet Transform-Based Satellite Image Resolution Enhancement" IEEE Trans. Geoscience And Remote Sensing Letters, Vol.7, No.5,May 2011.
[11] Ke, Wei-Ming, Chih-Rung Chen, and Ching-Te Chiu. "BiTA/SWCE: Image enhancement with bilateral tone adjustment and saliency weighted contrast enhancement." Circuits and Systems for Video Technology, IEEE Transactions on 21.3: pp.360-364, 2010.

[12] Chauhan, Ritu, and Sarita Singh Bhadoria. "An improved image contrast enhancement based on histogram equalization and brightness preserving weight clustering histogram equalization." Communication Systems and Network Technologies (CSNT), 2011 International Conference on. IEEE, 2011.

[13] Ghimire, Deepak, and Joonwhoan Lee. "Nonlinear transfer function-based local approach for color image enhancement." Consumer Electronics, IEEE Transactions on 57.2: pp.858-865, 2011.

[14] Josephus, ChelsySapna, and S. Remya. "Multilayered Contrast Limited Adaptive Histogram Equalization Using Frost Filter." Recent Advances in Intelligent Computational Systems (RAICS), IEEE, 2011.

[15] Lasmika, K. Raveendra, "Improving Quality of Satellite Image by Wavelet Transforming \& Morphological Filtering", International Journal of Innovative Research in Science, Engineering and Technology, ISSN: 23198753, Vol. 3, Issue 7, July 2014.

[16] E. Mohan, K.B. Jayarraman, U. Maheswaran, D. Sathiyaraj. G.Dhakshanamoorthi,"A novel aproach for satellite image resolution enhancement", International Journal Of Engineering And Advanced Technology (Ijeat) Issn:2249-8958, Volume-2, Issue-4, April 2013,Pp.112-114

[17] S.VENKATRAMANA, S. NARAYANA REDDY," A Novel Method To Improve Resolution Of Satellite Images Using DWT And Interpolation.” International Journal Of Advanced Research In Electrical, Electronics And Instrumentation Engineering, Vol.3, Issue 1,January 2014,Pp.6530-6536

[18] Sundaram, M., K. Ramar, N. Arumugam, and G. Prabin. "Histogram based contrast enhancement for mammogram images." In Signal Processing, Communication, Computing and Networking Technologies (ICSCCN), 2011 International Conference on, pp. 842-846. IEEE, 2011.

[19] Madhu S. Nair. And G. Raju "A fast and efficient color image enhancement method based on fuzzy logic and histogram". IEEE Trans Syst Man Cybern 1981;SMC-11(July (2013):494-501ss[6]

[20] Hasan Demirel And Gholamreza Anbarjafari,"Satellite Image Resolution Enhancement Using Dual Tree-Complex Wavelet Transform" IEEE Trans. Geoscience And Remote Sensing Letters, Vol.7, No.1, January 2010,Pp123-126. 